\title{
Commensurability Oscillations in One-Dimensional Graphene Superlattices
}

\author{
Martin Drienovsky, ${ }^{1}$ Jonas Joachimsmeyer, ${ }^{1}$ Andreas Sandner, ${ }^{1}$ Ming-Hao Liu (劉明豪), ${ }^{2,3,}$ Takashi Taniguchi, ${ }^{4}$ \\ Kenji Watanabe, ${ }^{4}$ Klaus Richter, ${ }^{3}$ Dieter Weiss, ${ }^{1}$ and Jonathan Eroms, ${ }^{1, *}$ \\ ${ }^{1}$ Institute of Experimental and Applied Physics, University of Regensburg, D-93040 Regensburg, Germany \\ ${ }^{2}$ Department of Physics, National Cheng Kung University, Tainan 70101, Taiwan \\ ${ }^{3}$ Institute of Theoretical Physics, University of Regensburg, D-93040 Regensburg, Germany \\ ${ }^{4}$ National Institute for Materials Science, 1-1 Namiki, Tsukuba 305-0044, Japan
}

(Received 30 January 2018; published 11 July 2018)

\begin{abstract}
We report the experimental observation of commensurability oscillations (COs) in 1D graphene superlattices. The widely tunable periodic potential modulation in $\mathrm{hBN}$-encapsulated graphene is generated via the interplay of nanopatterned few-layer graphene acting as a local bottom gate and a global Si back gate. The longitudinal magnetoresistance shows pronounced COs when the sample is tuned into the unipolar transport regime. We observe up to six CO minima, providing evidence for a long mean free path despite the potential modulation. Comparison to existing theories shows that small-angle scattering is dominant in $\mathrm{hBN} / \mathrm{graphene} / \mathrm{hBN}$ heterostructures. We observe robust COs persisting to temperatures exceeding $T=150 \mathrm{~K}$. At high temperatures, we find deviations from the predicted $T$ dependence, which we ascribe to electron-electron scattering.
\end{abstract}

DOI: 10.1103/PhysRevLett.121.026806

Due to its high intrinsic mobility [1], graphene is an ideal material for exploring ballistic phenomena. Both suspended graphene [2,3] and graphene/hexagonal boron nitride $(\mathrm{hBN})$ heterostructures $[4,5]$ were employed to demonstrate integer and fractional quantum Hall effects [6-9], conductance quantization [10,11], cyclotron orbits [12-15], and ballistic effects at $p-n$ junctions [16-18], all requiring high mobility.

In particular, several fascinating observations have been made in moiré superlattices in graphene/hBN heterostructures. In addition to magnetotransport signatures [19-21] of the fractal energy spectrum predicted by Hofstadter [22], Krishna Kumar et al. recently reported robust $1 / B$ periodic oscillations persisting to above room temperature [23]. Those oscillations were ascribed to band conductivity in superlattice-induced minibands, where the group velocity in those minibands enters into the magnetoconductance. Here, the oscillation period is independent of the carrier density and set only by the lattice spacing $a$ via $\Phi_{0} / \Phi$, where $\Phi_{0}$ is the magnetic flux quantum and $\Phi$ the flux through one superlattice unit cell.

However, the archetypal effect where a superlattice potential leads to magnetoresistance oscillations due to miniband conductivity, namely Weiss or commensurability oscillations (CO) [24], has not yet been demonstrated in graphene, owing to the challenging task of combining high-mobility graphene and a weak nanometer-scale periodic potential. Those oscillations arise due to the interplay between the cyclotron orbits of electrons in a high magnetic field and the superlattice potential. For a $1 \mathrm{D}$ modulation, pronounced $1 / B$-periodic oscillations in the magnetoresistance $R_{x x}$ are observed, with minima appearing whenever the cyclotron diameter $2 r_{C}$ is a multiple of the lattice period $a$, following the relation

$$
2 r_{C}=\left(\lambda-\frac{1}{4}\right) a,
$$

with $\lambda$ being an integer. This intuitive picture was confirmed in Beenakker's semiclassical treatment [25]. Quantum mechanically, without modulation and in a high magnetic field, Landau levels are highly degenerate in the quantum number $k_{y}$. The superlattice potential lifts the degeneracy and introduces a miniband dispersion $E_{N}\left(k_{y}\right)$ into each Landau level. The miniband width oscillates with both $1 / B$ and energy, and flat bands appear whenever Eq. (1) is fulfilled. Therefore, the group velocity $v_{g}=\partial E_{N} / \partial k_{y}(1 / \hbar)$ also oscillates, leading to magnetoconductance oscillations [26,27]. Those oscillations persist to higher temperatures than Shubnikov-de Haas oscillations ( $\mathrm{SdHO})$, since the band conductivity survives thermal broadening of the density of states. In contrast to the oscillations in Ref. [23], the COs depend on the electron density $n$ through $r_{C}=\hbar \sqrt{4 \pi n /\left(g_{s} g_{v}\right)} / e B$, where $g_{s}\left(g_{v}\right)$ is the spin (valley) degeneracy. The commensurability condition in Eq. (1) also holds in the case of graphene [28,29]. What is different, though, is the Landau level spectrum, which is equidistant in the case of a conventional 2DEG but has a square-root dependence in the case of the Dirac fermions in graphene $[30,31]$. This has been predicted to also modify the COs [28,29,32]. Notably, Matulis and Peeters calculated very robust COs in the quasiclassical region of small fields that should persist up to high temperatures [28]. 
Here we employ a patterned few-layer graphene backgating scheme $[33,34]$ to demonstrate clear-cut COs of both Dirac electrons and holes in high-mobility graphene, subjected to a weak unidirectional periodic potential. Contrary to hBN/graphene moiré lattices, where lattice parameters are set by the materials properties of graphene and $\mathrm{hBN}$, this enables us to define an arbitrary superlattice geometry and strength. As the usual technique of placing a metallic grating with nanoscale periodicity fails due to the poor adhesion of metal to the atomically smooth and inert $\mathrm{hBN}$ surface, we resort to including a patterned bottom gate (PBG) consisting of few-layer graphene (FLG) carrying the desired superlattice pattern into the usual van der Waals stacking and edge-contacting technique [5]. The hBN/ graphene/hBN stack is assembled on top of the PBG. Importantly, the bottom hBN layer has to be kept very thin $(<15 \mathrm{~nm})$ to impose the periodic potential effectively onto the unpatterned graphene sheet. For the PBG, we exfoliated a FLG sheet (3 to 4 layers) onto an oxidized, highly $p$-doped silicon wafer which served as a uniform global back gate in the measurements. The FLG sheet was patterned into the desired shape by electron beam lithography and oxygen plasma etching. This approach exploits the atomic flatness of FLG, which makes it a perfect gate electrode for 2D-material heterostructures that can be easily etched into various shapes, e.g., 1D or 2D superlattices, split gates, collimators [35], or lenses [36], and allows for nanoscale manipulation of the carrier density. Figure 1(a) shows the AFM image of an 80-nm-stripe lattice used for the fabrication of sample $B$, discussed below. The hBN/ graphene/hBN stack was deposited onto the PBG, and a mesa was defined by reactive ion etching [Fig. 1(b)]. We used a sequential etching method, employing $\mathrm{SF}_{6}$ [37], $\mathrm{O}_{2}$, and $\mathrm{CHF}_{3} / \mathrm{O}_{2}$ processes, in order to avoid damage to the thin hBN bottom layer covering the PBG (see the Supplemental Material [38] for details). Edge contacts of evaporated $\mathrm{Cr} / \mathrm{Au}(1 \mathrm{~nm} / 90 \mathrm{~nm})$ were deposited after reactive ion etching of the contact region and a brief exposure to oxygen plasma. More details on the fabrication are reported elsewhere [34].

The combined action of PBG and the global gate is sketched in Fig. 1(c). The PBG partially screens the electric field lines emerging from the Si back gate. The latter therefore controls the carrier type and density in the regions between stripes (labeled $n$ ), whereas the PBG itself controls primarily those directly above the stripes (labeled $n^{\prime}$ ). A typical charge-carrier density profile for a weak potential modulation in the unipolar transport regime is shown atop. Hence, tuning both gates separately, we can generate unipolar or bipolar potential modulation on the nanoscale.

Transport measurements were performed in a helium cryostat at temperatures between 1.4 and $200 \mathrm{~K}$ and in perpendicular magnetic fields between 0 and $10 \mathrm{~T}$ using low-frequency lock-in techniques at a bias current of $10 \mathrm{nA}$. We present data from two samples $(A$ and $B)$ with
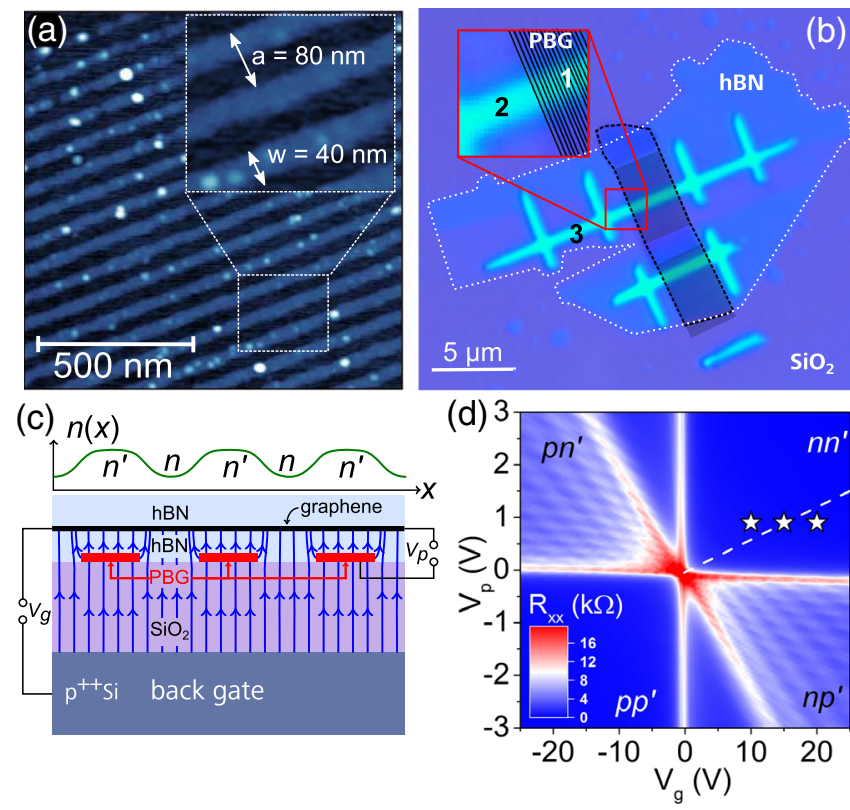

FIG. 1. Sample geometry and characteristics. (a) AFM image of the PBG of sample $B$. (b) hBN/graphene/hBN heterostructure on top of a few-layer graphene patterned bottom gate after mesa etching, before contact deposition (sample $B, \mathrm{PBG}$ outlined in black, lower hBN outlined in white). Labels 1, 2, and 3 denote the modulated, the unmodulated, and the reference area, respectively (see text). (c) Schematic longitudinal section of the sample geometry, showing the influence of the two independent gates on the graphene charge-carrier density in the case of a unipolar modulation. (d) Resistance map of sample $A$ as a function of gate voltages, $V_{p}$ (PBG) and $V_{g}$ (back gate) at $B=0$. The highly regular Fabry-Pérot pattern in the bipolar regions confirms the presence of identical barriers, forming a superlattice. The white dashed line in the $n n^{\prime}$ quadrant represents the $n=n^{\prime}$ configuration of the two independent gates. The configurations, marked by stars, will be addressed in the text and Fig. 2 .

1D-superlattice periods of $a_{A}=200 \mathrm{~nm}$ and $a_{B}=80 \mathrm{~nm}$, respectively. The PBGs of both samples, $A$ and $B$, consist of 19 and 40 stripes of few-layer graphene (thickness 3 to 4 layers), respectively. The thicknesses of the lower hBN, separating the graphene from the PBG, are $t_{A}=13 \mathrm{~nm}$ and $t_{B}=2 \mathrm{~nm}$, respectively, measured with AFM. Figure 1(d) displays the zero-field resistance of sample $A$. Using both gates, we can tune into the unipolar regime of comparatively low resistance (labeled $n n^{\prime}$ and $p p^{\prime}$ ), as well as the bipolar regime (labeled $p n^{\prime}$ and $n p^{\prime}$ ), where pronounced Fabry-Pérot oscillations appear [16,34,39-42]. Their regular shape proves the high quality and uniformity of the superlattice potential. The electrostatics of dual gated samples and different transport regimes were discussed, e.g., in Refs. [16,34,40,42].

Below, we focus on the unipolar regime, to obtain a weak and tunable 1D superlattice. This is the regime of the COs outlined above. Let us first discuss magnetotransport in sample $A$ with mobility $\mu \approx 55000 \mathrm{~cm}^{2} / \mathrm{V} \mathrm{s}$ (see the 


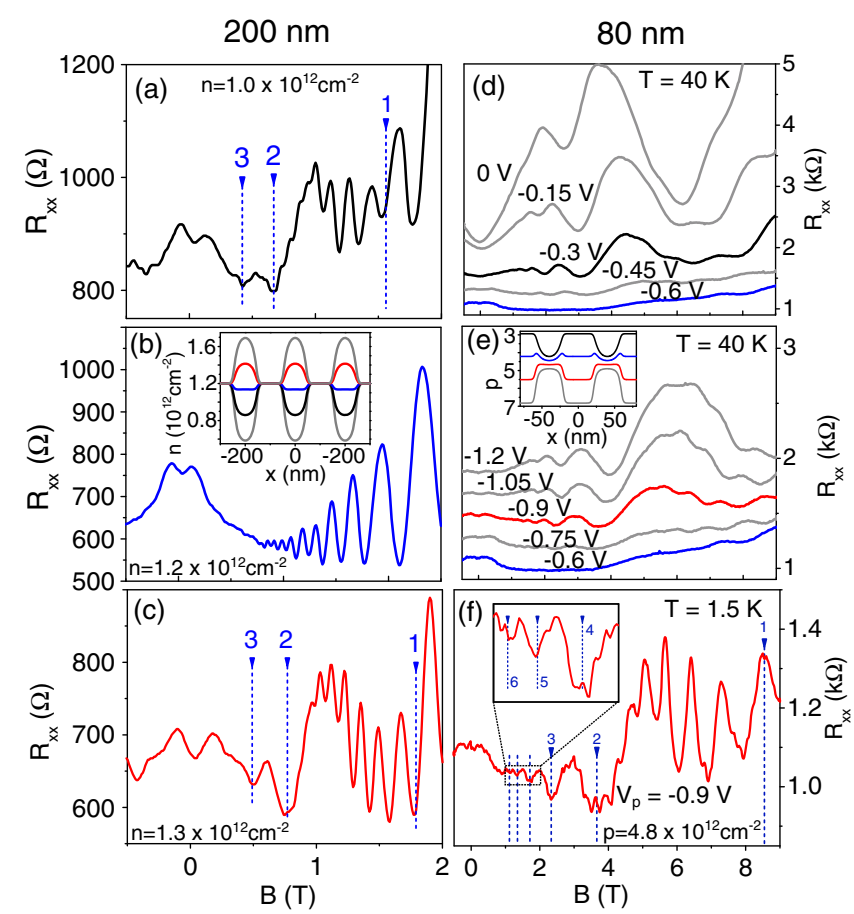

FIG. 2. Commensurability oscillations in graphene. (a)-(c) Magnetoresistance of sample $A\left(a_{A}=200 \mathrm{~nm}\right)$ at $T=1.5 \mathrm{~K}$ for fixed $V_{p}=0.9 \mathrm{~V}$ and $V_{g}=10,15,20 \mathrm{~V}$, respectively. The densities $n$ were extracted from SdHOs at higher fields. Vertical blue lines: Calculated flat band position [Eq. (1)]. The COs appear for weak modulation (a),(c) and disappear in the demodulated situation (b). The inset in (b) shows the calculated chargecarrier density profiles for blue $V_{g}=5 \ldots 25 \mathrm{~V}$, where situations (a)-(c) are represented by colors. (d),(e) Magnetoresistance of sample $B\left(a_{B}=80 \mathrm{~nm}\right)$ at $T=40 \mathrm{~K}$ and fixed $V_{g}=-25 \mathrm{~V}$, with (d) $V_{p}=0 \ldots-0.6 \mathrm{~V}$ and (e) $V_{p}=-0.6 \ldots-1.2 \mathrm{~V}$. (f) $T=1.5 \mathrm{~K}$, where minima up to $\lambda=6$ are resolvable. Inset in (e): 1D charge-carrier density distribution (units of $10^{12} \mathrm{~cm}^{-2}$ ) for $V_{p}=-0.3 \ldots-1.2 \mathrm{~V}$, where gate configurations in (d)-(f) are represented by colors.

Supplemental Material [38] for the determination of mobility in both samples). In Figs. 2(a)-2(c), we show three magnetic field sweeps, where we keep the PBG voltage fixed at $V_{p}=$ $0.9 \mathrm{~V}$ and tune the modulation strength by varying the backgate voltage $V_{g}$. The sweeps represent three different situations: (a) $n<n^{\prime}$, (b) $n \approx n^{\prime}$, and (c) $n>n^{\prime}$. The corresponding $V_{g}, V_{p}$ positions of the sweeps [(a)-(c)] are marked by stars in the $n n^{\prime}$ quadrant of Fig. 1(d). Moreover, the inset in Fig. 2(b) shows the corresponding charge-carrier density profiles that were calculated employing a 1D electrostatic model of the device, including a quantum capacitance correction $[40,43]$, but neglecting screening.

In Fig. 2(a), a weak, unipolar $\left(n<n^{\prime}\right)$ potential modulation is shown where the longitudinal resistance $R_{x x}$ exhibits well-pronounced peaks and dips prior to the emergence of SdHOs, appearing at slightly higher $B$ fields. The average charge-carrier density, extracted from SdHOs is $1.0 \times 10^{12} \mathrm{~cm}^{-2}$ for this particular gate configuration, yielding a mean free path $l_{f}=\hbar k_{F} \mu / e=0.64 \mu \mathrm{m}$. The expected flat band positions [Eq. (1)] are denoted by the blue vertical dotted lines, perfectly describing the experimentally observed minima. The dips are resolved up to $\lambda=3$, corresponding to a cyclotron orbit circumference of $2 \pi r_{C}=1.7 \mu \mathrm{m}$. This clearly confirms that ballistic transport is maintained over several periods of the superlattice.

At $V_{g}=15 \mathrm{~V}$ (Fig. 2(b)), $n \approx n^{\prime}$ holds (see inset in Fig. 2(b)). We still observe clear SdHOs, but the COs disappeared. The pronounced peak at $\sim \pm 0.16 \mathrm{~T}$ can be attributed to a magneto-size effect related to boundary scattering in ballistic conductors $[44,45]$ of width $W$. While in GaAs based 2DEGs, a ratio $W / r_{C} \approx 0.5$ is found, we extract $W / r_{C} \approx 1$ in accordance with previous studies on graphene [46].

Further increasing $V_{g}$ increases $n$ and switches the modulation on again $\left(n>n^{\prime}\right)$. The SdHOs in Fig. 2(c) yield an average $n=1.3 \times 10^{12} \mathrm{~cm}^{-2}$. Again, three minima appear at the expected flat band condition described by Eq. (1).

Let us turn to sample $B$, where we demonstrate COs in the $p$ regime. It has a short period of $a_{B}=80 \mathrm{~nm}$ and a bottom $\mathrm{hBN}$ flake of only $2 \mathrm{~nm}$ thickness, separating the PBG from the graphene. Figures 2(d) and 2(e) show the longitudinal resistance at high average hole densities $\left(-n=p \approx 4.5 \times 10^{12} \mathrm{~cm}^{-2}\right)$ as a function of the PBG voltage $V_{p}$ and the perpendicular $B$ field at fixed $V_{g}=$ $-25 \mathrm{~V}$ (Dirac point at $V_{g}=40 \mathrm{~V}$ ) and $T=40 \mathrm{~K}$. Here, $T$ was increased in order to damp the SdHOs for better resolution of the COs. The mobility of $\mu=30000 \mathrm{~cm}^{2} / \mathrm{V} \mathrm{s}$ and the rather large hole density $p$ give rise to a mean free path $l_{f} \approx 0.75 \mu \mathrm{m}$. We can resolve COs up to $\lambda=6$ [see Fig. 2(f) [47] ], corresponding to a cyclotron orbit circumference of $2 \pi r_{C}=1.4 \mu \mathrm{m}$, which is about twice $l_{f}$ in the average density range considered. At around $V_{p} \sim-0.6 \mathrm{~V}$, the COs disappear as the modulation potential becomes minimal [blue lines in Figs. 2(d) and 2(e), cf. charge-carrier density profile in the inset]. Here, $p \approx p^{\prime}$ holds and no COs are resolvable. This changes again at $V_{p}=-0.6 \ldots-1.2 \mathrm{~V}$ [Fig. 2(e)]. As the back-gate voltage is further increased, strong COs appear again, with the minima positions shifting according to the density dependence of Eq. (1) (see also the Supplemental Material [38] for a color map). The observation of clear-cut COs in density-modulated hole and electron systems for distinctively different superlattice periods highlights the suitability of graphene PBGs for imposing lateral potentials on graphene films.

As pointed out in the Introduction, theory predicted enhanced COs in graphene [28]. To check this and to compare theory and experiment, we apply the different prevailing theoretical models to describe $R_{x x}(B)$ for our sample. The amplitude of the COs is governed by the period $a$, the modulation amplitude $V_{0}$, and the Drude transport relaxation time $\tau_{p}$. Expressions for the additional band conductivity $\Delta \sigma_{y y}$ for $2 \mathrm{DEG}$ in Ref. [48] and 
graphene in Ref. [28] [see Eqs. (S1) and (S9) in the Supplemental Material [38], respectively] are linear in $B$ and tend to overestimate the $\mathrm{CO}$ amplitude at lower field. Mirlin and Wölfle [49] introduced anisotropic scattering to the problem by taking into account the small-angle impurity scattering, allowing for a high ratio of the momentum relaxation time $\tau_{p}$ to the elastic scattering time $\tau_{e}$ [see Eq. (S10) in the Supplemental Material [38]]. In this approach, both the damping of COs at lower fields and the modulation amplitudes of conventional 2DEGs are correctly described. For the graphene case, Matulis and Peeters employed the Dirac-type Landau level spectrum, as opposed to the parabolic 2DEG situation [28], leading to a modified expression. In their approach, only a single transport scattering time $\tau_{p}$ was included. The temperature dependence of the COs was treated in Refs. [48,50] for parabolic 2DEGs and in Ref. [28] for graphene. It is expected to exhibit a $x / \sinh (x)$-dependence, where $x=T / T_{c}$, with the critical temperature

$$
T_{c}=\frac{B e a}{4 \pi^{2} k_{B}} v_{F}
$$

Here, $k_{B}$ is the Boltzmann constant, and the difference between parabolic and linear dispersion is absorbed in the different Fermi velocities $v_{F}$.

To compare to the different theoretical models, we extracted the elastic scattering time $\tau_{e}=(80 \pm 10) \mathrm{fs}$ from the SdHO envelope [51] of a reference Hall bar (see the Supplemental Material [38] for details). With $\mu=30000 \mathrm{~cm}^{2} / \mathrm{Vs}$, we obtain the ratio $\tau_{p} / \tau_{e} \approx 7.4$, which emphasizes the importance of small-angle scattering in hBN-encapsulated graphene. The experimental (black) curve in Fig. 3(a) was taken at $T=40 \mathrm{~K}$, where the SdHOs are already visibly suppressed, but the amplitude of the COs is practically unchanged, allowing for a better
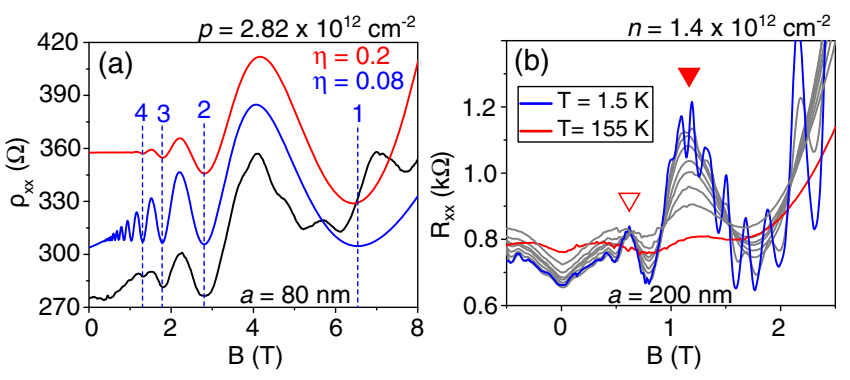

FIG. 3. (a) Comparison of different theoretical expressions with experiment at $V_{g}=-25 \mathrm{~V}, V_{p}=-0.8 \mathrm{~V}$, and $T=40 \mathrm{~K}$ (black curve). The blue curve represents the theory for graphene [28] with isotropic scattering and $\eta=0.08$. The red curve includes small-angle impurity scattering [49], using $\eta=0.2$, and matches the experiment well. The experimental curve does not show a pronounced minimum around $6 \mathrm{~T}$ due to strong SdHOs setting in. (b) Longitudinal magnetoresistance of sample A at $V_{g}=25 \mathrm{~V}$ and $V_{p}=0.9 \mathrm{~V}$ at different temperatures, from 1.5 to $155 \mathrm{~K}$. comparison to theory. We first compare our measurement to the graphene theory employing isotropic scattering only. Since the superlattice period $a_{B}=80 \mathrm{~nm}, \tau_{p}=0.59 \mathrm{ps}$, temperature $T=40 \mathrm{~K}$, and the average charge-carrier density $p=2.8 \times 10^{12} \mathrm{~cm}^{-2}$ are known, only the relative modulation strength $\eta=V_{0} / E_{F}$ remains as a fitting parameter. By fitting the theoretical expressions (for details, see the Supplemental Material [38]) to the CO peak at $\approx 4 \mathrm{~T}$ in Fig. 3(a), we obtain $\eta=0.08$. At lower fields, the experimentally observed oscillations decay much faster than the calculated ones. Inserting our sample parameters into the theory employing small-angle impurity scattering [49] [see Eq. (S10) in the Supplemental Material [38] ], again only $\eta=V_{0} / E_{F}$ remains as a free-fitting parameter, and we obtain the red trace using $\eta=0.2$. The fit describes the experimental magnetoresistance strikingly well, although the Dirac nature of the spectrum was not considered. The fits in Fig. 3(a) imply that including smallangle impurity scattering is essential for the correct description of encapsulated graphene.

Finally, we discuss the temperature dependence of COs in 1D modulated graphene. Figure 3(b) depicts a longitudinal resistance trace of sample $A$ at $n=1.4 \times 10^{12} \mathrm{~cm}^{2}$ different temperatures. The graph clearly demonstrates that the COs are much more robust than the SdHOs. While the latter are almost completely suppressed at $T=40 \mathrm{~K}$, the COs survive at least up to $T=150 \mathrm{~K}$ (sample $A$ ) and $T=$ $200 \mathrm{~K}$ (sample $B$ ). We analyze the temperature evolution of the first two CO peaks (marked by red triangles), using the connecting line between two adjacent minima as the bottom line to evaluate the height of the maximum in between. We adopt this procedure described by Beton et al. for a better comparison to experiments in GaAs [50]. The temperature dependence of the two peaks is shown in Fig. 4(a). Also shown are the corresponding data of sample $B$ (black symbols). The data for sample $B$ were extracted at much higher fields, due to the smaller lattice period and higher carrier density, leading to a weaker temperature dependence in Eq. (2). The expected temperature dependence (solid lines) $\left(T / T_{c}\right) / \sinh \left(T / T_{c}\right)$ $[28,48,50]$ clearly deviates from the experimental data points. For GaAs, $T$-dependent damping of the COs was so strong that the assumption of a $T$-independent scattering time was justified [50]. In graphene, the higher $v_{F}$ leads to a higher $T_{c}$, and therefore the COs persist to higher temperatures than in GaAs. Hence, we have to consider a $T$ dependence of the scattering time as well. Using the lowtemperature momentum relaxation time $\tau_{p}$, we first determine the modulation strength $\eta$, Then, using a fixed $\eta$, we extract the scattering time $\tau$ entering into the CO theory at elevated temperatures. The extracted times are plotted in Fig. 4(b) for both samples and two magnetic fields each, together with predictions for the electron-electron scattering time [52] and electron-phonon scattering time [53]. Clearly, at $T \gg 10 \mathrm{~K}, \tau$ deviates visibly from $\tau_{p}$, with $\tau_{e-e}$ being the relevant cutoff, while $\tau_{e-p h}$ is not important. 

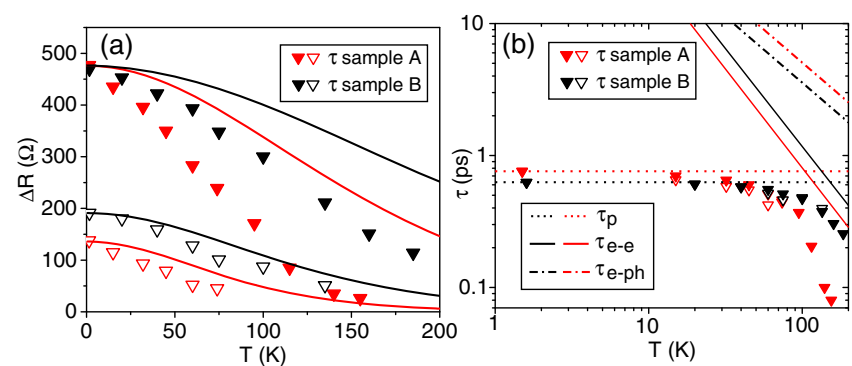

FIG. 4. (a) $T$ dependence of the $\mathrm{CO}$ peaks, marked by the red triangles in Fig. 3(b). Black triangles: Similar data for sample $B$ at density $p=2.82 \times 10^{12} \mathrm{~cm}^{-2}$. Solid lines: Expected $T$ dependence $x / \sinh (x)$, where $x=T / T_{c}$, with $T_{c}$ from Eq. (2). (b) Scattering times extracted for both samples, together with $\tau_{p}$ from $T=1.5 \mathrm{~K}$ and predictions for $\tau_{e-e}[52]$ and $\tau_{e-p h}$ [53].

This resembles the recently found observation window for hydrodynamic effects in graphene [54].

To conclude, we present the first experimental evidence of commensurability oscillations (COs) [24] for both electrons and holes in a hBN-encapsulated monolayer graphene subject to a $1 \mathrm{D}$ periodic potential. This was made possible through the combined action of a nanopatterned FLG bottom gate and a global Si back gate. Our approach allows tuning both carrier density and modulation strength independently in a wide range, and on the scale of a few tens of nanometers. The minima in $R_{x x}(B)$ are well described by the flat band condition [Eq. (1)]. The predicted strong temperature robustness of COs in graphene was qualitatively confirmed, but detailed comparison to existing theories emphasized the need for a description including anisotropic scattering of charge carriers in encapsulated graphene. Using data at elevated temperature, we could extract the $T$ dependence of the scattering time, pointing to electron-electron scattering as the high- $T$ cutoff for the $\mathrm{CO}$ amplitude.

Financial support by the Deutsche Forschungsgemeinschaft (DFG) within Programs No. GRK 1570 and No. SFB 689 (Projects No. A7 and No. A8) and Project No. Ri 681/ 13 "Ballistic Graphene Devices" and by the Taiwan Minister of Science and Technology (MOST) under Grant No. 107-2112-M-006-004-MY3 is gratefully acknowledged. Growth of hexagonal boron nitride crystals was supported by the Elemental Strategy Initiative conducted by the MEXT, Japan, and JSPS KAKENHI Grant No. JP15K21722. We thank J. Amann, C. Baumgartner, A. T. Nguyen, and J. Sahliger for their contribution towards the optimization of the fabrication procedure.

*jonathan.eroms@ur.de †minghao.liu@phys.ncku.edu.tw

[1] S. V. Morozov, K. S. Novoselov, M. I. Katsnelson, F. Schedin, D. C. Elias, J. A. Jaszczak, and A. K. Geim, Phys. Rev. Lett. 100, 016602 (2008).
[2] X. Du, I. Skachko, A. Barker, and E. Y. Andrei, Nat. Nanotechnol. 3, 491 (2008).

[3] K. Bolotin, K. Sikes, Z. Jiang, M. Klima, G. Fudenberg, J. Hone, P. Kim, and H. Stormer, Solid State Commun. 146, 351 (2008).

[4] C. R. Dean, A. F. Young, I. Meric, C. Lee, L. Wang, S. Sorgenfrei, K. Watanabe, T. Taniguchi, P. Kim, K. L. Shepard, and J. Hone, Nat. Nanotechnol. 5, 722 (2010).

[5] L. Wang, I. Meric, P. Huang, Q. Gao, Y. Gao, H. Tran, T. Taniguchi, K. Watanabe, L. Campos, D. Muller et al., Science 342, 614 (2013).

[6] A. F. Young, C. R. Dean, L. Wang, H. Ren, P. CaddenZimansky, K. Watanabe, T. Taniguchi, J. Hone, K. L. Shepard, and P. Kim, Nat. Phys. 8, 550 (2012).

[7] K. I. Bolotin, F. Ghahari, M. D. Shulman, H. L. Stormer, and P. Kim, Nature (London) 462, 196 (2009).

[8] X. Du, I. Skachko, F. Duerr, A. Luican, and E. Y. Andrei, Nature (London) 462, 192 (2009).

[9] J. I. A. Li, C. Tan, S. Chen, Y. Zeng, T. Taniguchi, K. Watanabe, J. Hone, and C. R. Dean, Science 358, 648 (2017).

[10] N. Tombros, A. Veligura, J. Junesch, M. H. D. Guimaraes, I. J. Vera-Marun, H. T. Jonkman, and B. J. van Wees, Nat. Phys. 7, 697 (2011).

[11] B. Terrés, L. A. Chizhova, F. Libisch, J. Peiro, D. Jörger, S. Engels, A. Girschik, K. Watanabe, T. Taniguchi, S. V. Rotkin, J. Burgdörfer, and C. Stampfer, Nat. Commun. 7, 11528 (2016).

[12] T. Taychatanapat, K. Watanabe, T. Taniguchi, and P. JarilloHerrero, Nat. Phys. 9, 225 (2013).

[13] M. Lee, J. R. Wallbank, P. Gallagher, K. Watanabe, T. Taniguchi, V. I. Fal'ko, and D. Goldhaber-Gordon, Science 353, 1526 (2016).

[14] A. Sandner, T. Preis, C. Schell, P. Giudici, K. Watanabe, T. Taniguchi, D. Weiss, and J. Eroms, Nano Lett. 15, 8402 (2015).

[15] R. Yagi, R. Sakakibara, R. Ebisuoka, J. Onishi, K. Watanabe, T. Taniguchi, and Y. Iye, Phys. Rev. B 92, 195406 (2015).

[16] P. Rickhaus, R. Maurand, M.-H. Liu, M. Weiss, K. Richter, and C. Schönenberger, Nat. Commun. 4, 2342 (2013).

[17] P. Rickhaus, M.-H. Liu, P. Makk, R. Maurand, S. Hess, S. Zihlmann, M. Weiss, K. Richter, and C. Schönenberger, Nano Lett. 15, 5819 (2015).

[18] G.-H. Lee, G.-H. Park, and H.-J. Lee, Nat. Phys. 11, 925 (2015).

[19] C. R. Dean, L. Wang, P. Maher, C. Forsythe, F. Ghahari, Y. Gao, J. Katoch, M. Ishigami, P. Moon, M. Koshino, T. Taniguchi, K. Watanabe, K. L. Shepard, J. Hone, and P. Kim, Nature (London) 497, 598 (2013).

[20] B. Hunt, J. Sanchez-Yamagishi, A. Young, M. Yankowitz, B. J. LeRoy, K. Watanabe, T. Taniguchi, P. Moon, M. Koshino, P. Jarillo-Herrero et al., Science 340, 1427 (2013).

[21] L. A. Ponomarenko, R. V. Gorbachev, G. L. Yu, D. C. Elias, R. Jalil, A. A. Patel, A. Mishchenko, A. S. Mayorov, C. R. Woods, J. R. Wallbank, M. Mucha-Kruczynski, B. A. Piot, P. M, I. V. Grigoreva, K. S. Novoselov, F. Guinea, V. I. Fal'ko, and A. K. Geim, Nature (London) 497, 594 (2013). [22] D. R. Hofstadter, Phys. Rev. B 14, 2239 (1976). 
[23] R. Krishna Kumar, X. Chen, G. H. Auton, A. Mishchenko, D. A. Bandurin, S. V. Morozov, Y. Cao, E. Khestanova, M. Ben Shalom, A. V. Kretinin, K. S. Novoselov, L. Eaves, I. V. Grigorieva, L. A. Ponomarenko, V. I. Fal'ko, and A. K. Geim, Science 357, 181 (2017).

[24] D. Weiss, K. Klitzing, K. Ploog, and G. Weimann, Europhys. Lett. 8, 179 (1989).

[25] C. W. J. Beenakker, Phys. Rev. Lett. 62, 2020 (1989).

[26] R. Gerhardts, D. Weiss, and K. v. Klitzing, Phys. Rev. Lett. 62, 1173 (1989).

[27] R. W. Winkler, J. P. Kotthaus, and K. Ploog, Phys. Rev. Lett. 62, 1177 (1989).

[28] A. Matulis and F. M. Peeters, Phys. Rev. B 75, 125429 (2007).

[29] M. Tahir, K. Sabeeh, and A. MacKinnon, J. Phys. Condens. Matter 19, 406226 (2007).

[30] K. S. Novoselov, A. K. Geim, S. V. Morozov, D. Jiang, M. I. Katsnelson, I. V. Grigorieva, S. V. Dubonos, and A. A. Firsov, Nature (London) 438, 197 (2005).

[31] Y. Zhang, Y.-W. Tan, H. Störmer, and P. Kim, Nature (London) 438, 201 (2005).

[32] R. Nasir, K. Sabeeh, and M. Tahir, Phys. Rev. B 81, 085402 (2010).

[33] J. Li, K. Wang, K. J. McFaul, Z. Zern, Y. Ren, K. Watanabe, T. Taniguchi, Z. Qiao, and J. Zhu, Nat. Nanotechnol. 11, 1060 (2016).

[34] M. Drienovsky, A. Sandner, C. Baumgartner, M.-H. Liu, T. Taniguchi, K. Watanabe, K. Richter, D. Weiss, and J. Eroms, arXiv:1703.05631.

[35] V. V. Cheianov and V. I. Fal'ko, Phys. Rev. B 74, 041403 (2006).

[36] M.-H. Liu, C. Gorini, and K. Richter, Phys. Rev. Lett. 118, 066801 (2017).

[37] F. Pizzocchero, L. Gammelgaard, B. S. Jessen, J. M. Caridad, L. Wang, J. Hone, P. Bøggild, and T. J. Booth, Nat. Commun. 7, 11894 (2016).

[38] See Supplemental Material at http://link.aps.org/ supplemental/10.1103/PhysRevLett.121.026806 for details of the etching procedure, additional experimental data, details on theories in GaAs and graphene, determination of scattering times, and details on the fitting to experimental data.
[39] A. F. Young and P. Kim, Nat. Phys. 5, 222 (2009).

[40] M. Drienovsky, F.-X. Schrettenbrunner, A. Sandner, D. Weiss, J. Eroms, M.-H. Liu, F. Tkatschenko, and K. Richter, Phys. Rev. B 89, 115421 (2014).

[41] C. Handschin, P. Makk, P. Rickhaus, M.-H. Liu, K. Watanabe, T. Taniguchi, K. Richter, and C. Schönenberger, Nano Lett. 17, 328 (2017).

[42] S. Dubey, V. Singh, A. K. Bhat, P. Parikh, S. Grover, R. Sensarma, V. Tripathi, K. Sengupta, and M. M. Deshmukh, Nano Lett. 13, 3990 (2013).

[43] M.-H. Liu, Phys. Rev. B 87, 125427 (2013).

[44] T. J. Thornton, M. L. Roukes, A. Scherer, and B. P. Van de Gaag, Phys. Rev. Lett. 63, 2128 (1989).

[45] C. Beenakker and H. van Houten, Solid State Phys. 44, 1 (1991).

[46] S. Masubuchi, K. Iguchi, T. Yamaguchi, M. Onuki, M. Arai, K. Watanabe, T. Taniguchi, and T. Machida, Phys. Rev. Lett. 109, 036601 (2012).

[47] Fine features in Fig. 2(f) are presumably due to mesoscopic fluctuations.

[48] F. M. Peeters and P. Vasilopoulos, Phys. Rev. B 46, 4667 (1992).

[49] A. D. Mirlin and P. Wölfle, Phys. Rev. B 58, 12986 (1998).

[50] P. H. Beton, P. C. Main, M. Davison, M. Dellow, R. P. Taylor, E. S. Alves, L. Eaves, S. P. Beaumont, and C. D. W. Wilkinson, Phys. Rev. B 42, 9689 (1990).

[51] M. Monteverde, C. Ojeda-Aristizabal, R. Weil, K. Bennaceur, M. Ferrier, S. Guéron, C. Glattli, H. Bouchiat, J. N. Fuchs, and D. L. Maslov, Phys. Rev. Lett. 104, 126801 (2010).

[52] R. K. Kumar, D. A. Bandurin, F. M. D. Pellegrino, Y. Cao, A. Principi, H. Guo, G. H. Auton, M. B. Shalom, L. A. Ponomarenko, G. Falkovich, K. Watanabe, T. Taniguchi, I. V. Grigoreva, L. S. Levitov, M. Polini, and A. K. Geim, Nat. Phys. 13, 1182 (2017).

[53] E. H. Hwang and S. Das Sarma, Phys. Rev. B 77, 115449 (2008).

[54] D. Y. H. Ho, I. Yudhistira, N. Chakraborty, and S. Adam, Phys. Rev. B 97, 121404 (2018). 